\title{
Development of Wheel-less Snake Robot with Two Distinct Gaits and Gait Transition Capability
}

\author{
M. H. A. Majid \\ M. R. Khan \\ S. N. Sidek \\ Department of Mechatronics Engineering, International Islamic University Malaysia, Kuala Lumpur, Malaysia
}

\begin{abstract}
Snake robots are mostly designed based on single mode locomotion. However, single mode gait most likely could not work effectively when the robot is subject to an unstructured working environment with different measures of terrain complexity. As a solution, mixed mode locomotion is proposed in this paper by synchronizing two types of gaits known as serpentine and wriggler gaits used for non-constricted and narrow space environments, respectively, but for straight line locomotion only. A gait transition algorithm is developed to efficiently change the gait from one to another. This study includes the investigation on kinematics analysis followed by dynamics analysis while considering related structural constraints for both gaits. The approach utilizes the speed of the serpentine gait for open area locomotion and exploits the narrow space access capability of the wriggler gait. Hence, it can increase motion flexibility in view of the fact that the robot is able to change its mode of locomotion according to the working environment.
\end{abstract}

Keywords: Bio-inspired robots, robotics, robot dynamics, robot kinematics, robotic mechanical systems.

\section{Introduction}

Successful snake robot design means the snake robot has motion flexibility as possessed by a real snake. The only way to improve motion flexibility of the snake robot is to incorporate as many gaits as possible to suit varieties of working spaces and the robot should effectively change its gait to adapt to the change of the environment. Snake robot studied by Hirose and Umetani ${ }^{[1]}$ ignited a momentum in snake robot research. In general, there are four most common gaits found in the biological snake namely serpentine, concertina, sidewinding and rectilinear, which are currently adapted as snake robot locomotion gaits ${ }^{[2]}$.

Serpentine is known to be the most prominent and simplest form of snake robot gait inspired from nature. It is a sinusoidal form of locomotion and conventionally generated by approximating a serpenoid curve ${ }^{[3]}$. Recently, some modifications have been made to improve the workability of the conventional serpentine gait. The serpentine gait with variable bending angles as observed in natural snakes has been introduced into the snake robot and achieved a better locomotion efficiency ${ }^{[4]}$. Conventional serpentine model is based on fixed parameters while a modified serpenoid curve was developed in [5] which used variable parameters to avoid side slippage. In addition, vertical plane locomotion of the serpentine gait known as traveling wave locomotion was also studied in $[6,7]$. It has a similar model to the serpentine gait except the motion is generated in the vertical direction.

Sidewinding is another type of snake gait usually observed in the desert snakes. A novel kinematics model of the sidewinding gait locomotion is generated by repetitive traveling waves of the bending mechanism by using an algorithm based on the backbone curve model ${ }^{[8]}$. In the latest work, stability conditions were identified for sidewinders on a slope and a solution for a minimum aspect ratio of

Manuscript received March 12, 2013; revised August 23, 2013 the sidewinding pattern needed to maintain stability was presented $^{[9]}$. It is also important to note that the sidewinding locomotion involves sinus lifting where the snake robot lifts some parts of its body above the ground and switches the grounded parts of its body dynamically ${ }^{[10]}$.

Rectilinear locomotion is known as the slowest among the four primary types of snake robot gaits. Few works are found related to this type of locomotion. A snake robot that used shape memory alloy (SMA) as actuators and moved in a similar way to rectilinear locomotion was introduced in [11]. However, this snake robot moves with a very low average speed of $0.025 \mathrm{~cm} / \mathrm{s}$. Furthermore, the kinematic and dynamic models for a rectilinear gait have been developed considering the changes of mechanism topology over the course of the gait ${ }^{[12]}$. A simplified model of rectilinear gait was proposed in [13] where a simple two-mass model was employed to analyze the motion and the damping coefficient was varied to change speed.

Concertina is another type of snake robot locomotion gait and it is normally used for climbing purposes ${ }^{[14]}$. Its mathematical model and optimum conditions to reduce actuation torque during the locomotion were also investigated. The development of the kinematics model of concertina gait used similar locomotion of real snakes for horizontal motion is among one of the latest works. A new dynamic curve for modeling different parts of the snake robot was also presented in [15].

There are other gaits that have been scrutinized apart from the main four types previously described. For example, a new gait called forward head serpentine (FSH) was introduced in [16] while optimization of the FSH was presented in [17]. The FSH ensures that the head of snake robot always remains in the general direction of motion for easier sensing of obstacles in the environment and significantly enhances the easiness of the information processing. A gait is called a helical gait which defines as the locomotion of a snake-like robot convoluting around a pole or inside 
a pipe to propel itself forward ${ }^{[18]}$. Recently, a snake robot was designed with the capability of moving in a small diameter pipe. For example, a KAEROT-snake IV robot could move in a sequence of holding motions as well as with a sinusoidal mode motion inside a vertical or horizontal small diameter pipe ${ }^{[19]}$. Another locomotion gait used inside pipe is called helical rolling motion and it makes use of mathematical continuum curve model for body shape control ${ }^{[20]}$. Pedal wave locomotion with steering capability for narrow space application was proposed and consequently tested as continuous snake robot model in [21].

Research on mixed mode locomotion is slowly progressing against unique locomotion. In order to support the multimode locomotion, a robot must have at least four capabilities $^{[22]}$. First, it must be able to perform different locomotion modes. Second, it must be able to recover from unexpected locomotion failures. Third, it must be able to shift from one mode to another and finally, it must be able to choose the correct mode for the correct environment. A snake robot capable of anguilliform swimming like sea-snakes and lampreys in water and lateral undulatory locomotion like a snake on ground were found in [23]. The latest work proposed on snake robot can realize both the serpentine and the rectilinear motions, and the fusion of the two motions resulted in a higher adaptability to the environment ${ }^{[24]}$. In terms of the snake robot structure for different environment locomotions, self-reconfigurable modular robots could be found in the literature to produce multiple types of locomotion gaits ${ }^{[25-27]}$. These robots used universal joint to move in many environments successfully but the prototype structure does not follows the actual snake. Universal joint is used because it provides good access for the 3-dimensional (3D) locomotion.

In this paper, two distinct gaits namely serpentine used for open space locomotion and wriggler used for narrow space locomotion are synchronized together and implemented on a snake robot prototype. These two gaits are chosen to accommodate the most common environment faced by the snake robots. As for example, narrow space such as a conduit, pipe, collapse building and bushes, apart from open environment are the most common types of workspace faced by the snake robot in real environment. In this work, we try to mimic a real snake structure in terms of friction elements (non-wheel) and bone structure in designing the snake robot prototype. Detail discussion on prototype design can be found in Section 6 .

\section{Kinematics modeling}

Fig. 1 illustrates the gait switching the proposed snake robot deploys to adapt to the two different types of environments. From the figure, it is clearly evidenced why the serpentine gait is not suitable for narrow space compared to the wriggler gait. The lateral width of the serpentine gait is larger compared to the wriggler and thus is not suitable for narrow space locomotion. In this section, two kinematics models of the gait are discussed in detail.

In this research, open space is defined as a space with dimension larger than the diameter hull of the serpentine gait while narrow space is defined as a space with dimension smaller than the diameter hull of the serpentine gait and larger than the diameter hull of the wriggler gait.

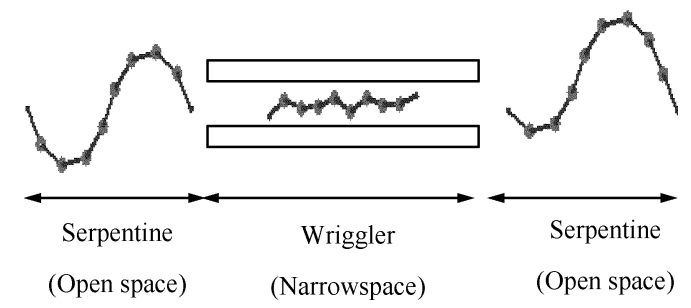

Fig. 1 Illustration of environment adaptation gait switching

\subsection{Kinematics of serpentine gait}

The first type of gait considered in this paper is a discrete serpentine gait ${ }^{[28]}$. The gait is the most common gait found in the literature where the joint angle $\phi$ is defined as a change of a sequence of absolute angles $\theta^{\prime}$ s and given as

$$
\phi_{i}=\theta_{i+1}-\theta_{i}=\alpha \sin \left(i \beta+\frac{\beta}{2}\right)+\gamma
$$

where parameters $\alpha, \beta$ and $\gamma$ are given by

$$
\begin{aligned}
& \alpha=a\left|\sin \left(\frac{\beta}{2}\right)\right| \\
& \beta=\frac{b}{n} \\
& \gamma=-\frac{c}{n} .
\end{aligned}
$$

From (1), the shape of the serpentine gait is in sinusoidal form with amplitude of $\alpha$ and with bias of $\gamma$. Relative angles which are adjacent to each other have phase difference of $\beta$. The values of $\alpha, \beta$ and $\gamma$ could be controlled by adjusting the values of $a, b$ and $c$, respectively, and consequently the shape of serpentine gait could change. Once the relative angles of the joints are known, it positions could be calculated geometrically on Cartesian coordinates as shown in Fig. 2. From Fig. 2, the $x$ and $y$ positions of the joint could be tracked by using the following equations.

$$
\begin{aligned}
& x_{k}=x_{0}+\sum_{k=1}^{n} 2 l \cos \theta_{k} \\
& y_{k}=y_{0}+\sum_{k=1}^{n} 2 l \sin \theta_{k}
\end{aligned}
$$

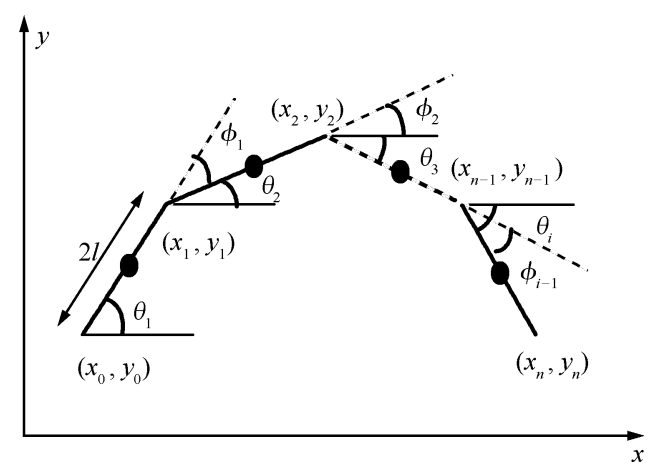

Fig. 2 Schematic of serpentine gait model 
where $\left(x_{0}, y_{0}\right)$ represents the tail coordinates of the snake robot and $\theta_{k}$ is the absolute angle for the $i$-th link which has equivalent length of $2 l$. Thus, once the absolute angles are known, using (5) and (6), the tracking of the snake robot position on the $x-y$ plane is possible. Different from robotic manipulator which has a fixed base at $\left(x_{0}, y_{0}\right)$, the snake robot tail is moving according to the body movement and thus the terms are not eliminated from the velocity expression. Differentiating (5) and (6) once, the $x$ and $y$ components of velocities are obtained as follows.

$$
\begin{aligned}
& \dot{y}_{i}=\dot{y}_{0}+2 l \sum_{i=1}^{i-1} \dot{\theta}_{k} \cos \theta_{k} \\
& \dot{x}_{i}=\dot{x}_{0}+2 l \sum_{k=1}^{i-1} \dot{\theta}_{k} \sin \theta_{k} .
\end{aligned}
$$

\subsection{Kinematics of wriggler gait}

The second type of gait considered in this study is the wriggler gait published in our previous paper ${ }^{[29]}$. The gait has been specifically modeled using cross link member to suit narrow space application. However, in the prototype design, the cross link member is considered virtually such that the link is not visible as cross link but its kinematics is retained. The derivation of the inverse kinematics model of the gait based on cross link is shown in Fig. 3 with length $2 l$ and width $2 w$. From Fig. 3 , the first joint position $\left(h_{1}\right.$, $k_{1}$ ) is determined geometrically using (9) and (10).

$$
\begin{aligned}
& h_{1}=r \cos \varphi_{1}+x_{1} \\
& k_{1}=r \sin \varphi_{1}+y_{1} .
\end{aligned}
$$

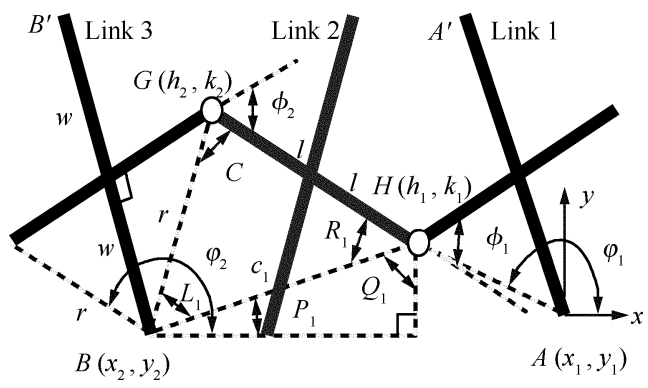

Fig. 3 Schematic of wriggler gait model

The length of segment $r$ and segment $c_{1}$ are given by

$$
\begin{aligned}
& r=\sqrt{l^{2}+w^{2}} \\
& c_{1}=\sqrt{\left(h_{1}-x_{2}\right)^{2}+\left(k_{1}-y_{2}\right)^{2}} .
\end{aligned}
$$

Using the cosine rule for the triangle formed by the joint $H\left(h_{1}, k_{1}\right)$, the second joint $G\left(h_{2}, k_{2}\right)$ and the second scale position $B\left(x_{2}, y_{2}\right)$, the three angles $R_{1}, C_{1}$ and $L_{1}$ can be calculated as follows.

$$
\begin{aligned}
& R_{1}=\cos ^{-1}\left(\frac{4 l^{2}+c_{1}^{2}-r^{2}}{4 l c_{1}}\right) \\
& C_{1}=\cos ^{-1}\left(\frac{r^{2}+c_{1}^{2}-4 l^{2}}{2 r c_{1}}\right) \\
& L_{1}=\cos ^{-1}\left(\frac{4 l^{2}+r^{2}-c_{1}^{2}}{4 l r}\right) .
\end{aligned}
$$

Using $c_{1}$ as the hypotenuse of the right triangle $A D H$, the two angles are obtained as

$$
\begin{aligned}
& Q_{1}=\tan ^{-1}\left(\frac{h_{1}-x_{2}}{k_{1}-y_{2}}\right) \\
& P_{1}=\tan ^{-1}\left(\frac{k_{1}-y_{2}}{h_{1}-x_{2}}\right) .
\end{aligned}
$$

The relationship between the first joint angle, $\phi_{1}$ and the first position of scales, $\varphi_{1}$ is

$$
\phi_{1}=\varphi_{1}+R_{1}+P_{1}-\frac{5 \pi}{4}
$$

and the second joint angle is

$$
\phi_{2}=L_{1}+Q_{1}+\frac{\pi}{2} .
$$

All the subsequent angles could be derived in a similar manner. The length $l$ and $w$ could be set to any value. However, to maintain the compatibility of the structure for narrow space application, $l$ is set to be equal to $w$. Thus from the structure, it is clear that all joint angles of the robot are limited within the range of

$$
-\frac{\pi}{4} \leqslant \phi \leqslant \frac{\pi}{4}
$$

As stated earlier, (18) and (19) constitute the inverse kinematics of the system. It is difficult to obtain forward kinematics equation straight away from the inverse kinematics. However, to arrive at the forward kinematics equations, curve fitting approximation method could be used by plotting the scale position against the joint angle within the range given in (20) and applying the curve fitting method.

The mechanism of wriggler gait movement is such that the scale at points $A$ and $B^{\prime}$ undergo dig-in effect while points $A^{\prime}$ and $B$ are free to move with respect to points $A$ and $B^{\prime}$, respectively. At the next step of motion, points $A^{\prime}$ and $B$ undergo dig-in effect while points $A$ and $B^{\prime}$ are free to move with respect to points $A$ and $B^{\prime}$, respectively. This sequence is repeated continuously to produce forward locomotion with the aid of the scales located at the belly of the snake robot.

\subsection{Gait kinematics constraints}

The two gaits discussed earlier have distinct kinematics structures. The kinematic model of a serpentine gait is based on straight link structure while the kinematic model of a wriggler gait is developed from the cross link structure. A kinematics constraint for both gaits under consideration is a key concept in designing a snake robot prototype. In other words, the prototype developed must satisfy the gait design criteria in terms of kinematics and link geometry.

\section{Dynamics modeling}

The equation of motion of the snake robot is similar to a robotic manipulator except the body is not fixed to the ground and it is subjected to the friction force between the surface and the body contact points. For a planar snake 
robot model with $n$ number of links where its potential energy is assumed to be zero, the general form of equation of motion based on energy method is given by [30]

$$
\frac{\mathrm{d}}{\mathrm{d} t} \frac{T}{\partial \dot{q}_{i}}-\frac{\partial T}{\partial q_{i}}+\frac{\partial R}{\partial \dot{q}_{i}}=Q_{i}, \quad i=1, \cdots, n
$$

where $q$ is the generalized coordinate. The kinetic energy $T$ and dissipative energy $R$ are given by

$$
\begin{aligned}
& T=\frac{1}{2} \sum_{i=1}^{n}\left[m_{i}\left(\dot{x}_{i}^{2}+\dot{y}_{i}^{2}\right)+I_{i} \ddot{\theta}_{i}^{2}\right] \\
& R=\frac{1}{2} \sum_{i=1}^{n}\left[D_{x y}\left(\dot{x}_{i}^{2}+\dot{y}_{i}^{2}\right)+d_{\theta} \dot{\theta}_{i}^{2}\right]+\sum_{i=2}^{n} D_{P} \dot{P}_{i} .
\end{aligned}
$$

Equations (22) and (23) can be simplified as

$$
\begin{aligned}
T & =\frac{1}{2} \dot{q}^{\mathrm{T}} M(\theta) \dot{q} \\
R & =\frac{1}{2} \dot{q}^{\mathrm{T}} D(\theta) \dot{q}
\end{aligned}
$$

where $m_{i}$ is mass of link $i, I_{i}$ is moment of inertia of link $i, D_{x y}$ and $D_{\theta}$ are the coefficients of the translational and rotational motions, respectively. $D_{P}$ is the coefficient of the friction in the robot joint. From (21), the dynamic model can be derived as

$$
M(\theta) \ddot{q}+C(\dot{\theta}, \theta) \dot{\theta}+D(\theta) \dot{q}=\left[\begin{array}{l}
\tau \\
0
\end{array}\right]
$$

where $\theta=\left[\theta_{1}, \theta_{2}, \cdots, \theta_{n}\right]^{\mathrm{T}} \in \mathbf{R}^{n}, \tau \in \mathbf{R}^{n-1}$ are joint torques, $M \in \mathbf{R}^{n+2 \times n+2}$ is an inertia matrix, $C \in \mathbf{R}^{n+2 \times n}$ is a centrifugal coefficient matrix, $D \in \mathbf{R}^{n+2 \times n+2}$ is a frictional coefficient matrix.

\section{Actuators torque}

Using the dynamic equation (26), the desired torque for each joint is calculated. Fig. 4 illustrates the overall process for calculating the desired joint torques.

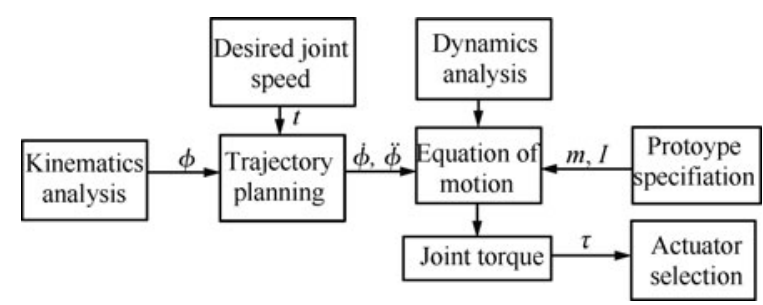

Fig. 4 Flow of joint's torque computation

Trajectory planning is used to determine the angular speed and angular acceleration of the joints. At least a set of data for each initial and final positions should be known for trajectory computation. These values are obtained from the kinematics analysis discussed earlier. An acceptable simulation time, $t$ is selected based on the desired actuator speed. A simple third-order polynomial trajectory planning as in (27) is used to generate smooth trajectory and used for torque computation in actuator selection. Since our method of control is purely based on kinematics, the jerky motion as a result from cubic polynomial trajectory planning is ignored and smoother trajectory will be considered for complete control system in the future study.

Thus, at least four initial conditions are required to solve for the unknown coefficients $c$ and consequently to obtain the expression for angular velocity and angular acceleration. The initial and final joint positions are known from kinematics analysis and the initial and final velocities are assumed to be zero as follows.

$$
\begin{aligned}
& \phi(t)=c_{0}+c_{1} t+c_{2} t^{2}+c_{3} t^{3} \\
& \phi\left(t_{i}\right)=\phi_{i}, \phi\left(t_{f}\right)=\phi_{f} \\
& \dot{\phi}\left(t_{i}\right)=0, \dot{\phi}\left(t_{f}\right)=0 .
\end{aligned}
$$

Once the torque for each joint of each gait is known, a comparison between the both gaits is made to select the largest torque for each joint. The results of joint torque computation are shown in Table 1.

Table 1 shows the comparison of joint torques obtained from simulation for both gaits used in the study. All parameter values in the simulation were used in the prototype fabrication. The number of joints used is eight $(i=8)$ connecting nine segments $(n=9)$. The prototype design is discussed in Section 6 .

Table 1 Comparison of joint's torque between serpentine and wriggler gaits

\begin{tabular}{ccc}
\hline $\begin{array}{c}\text { Joint } \\
\text { number }\end{array}$ & \multicolumn{2}{c}{ Maximum torque $(\mathrm{N} \cdot \mathrm{m})$} \\
\hline 1 & Serpentine gait & Wriggler gait \\
2 & 1.550 & 1.180 \\
3 & 1.280 & 0.450 \\
4 & 1.120 & 0.880 \\
5 & 1.020 & 2.530 \\
6 & 0.890 & 1.180 \\
7 & 0.630 & 0.450 \\
8 & 0.310 & 0.780 \\
\hline
\end{tabular}

From Table 1, in order to standardize the actuators size, the maximum torque of $2.53 \mathrm{~N} \cdot \mathrm{m}$ is selected and thus, the actuators chosen must have torque similar or greater than this value to ensure the actuators would provide enough torque to execute both gaits under consideration. Figs. 5 and 6 show the simulated power consumptions with respect to time for the serpentine gait and the wriggler gait, respectively for one step motion. It shows that the serpentine gait consumes more power compared to the wriggler gait because each joint in the serpentine gait rotates in the larger range of angle for each of the locomotion step.

\section{Gait transition algorithm}

After the kinematic models of the gaits are obtained, the gait switching algorithm is developed. The main purpose of the gait switching algorithm is to ensure that the snake robot would switch its gaits efficiently from one to another and at the same time maintaining the desired shape of the gait and the direction of locomotion. The algorithm for gait exchange is used between the two gaits either from serpen- 
tine to wriggler or vice versa.

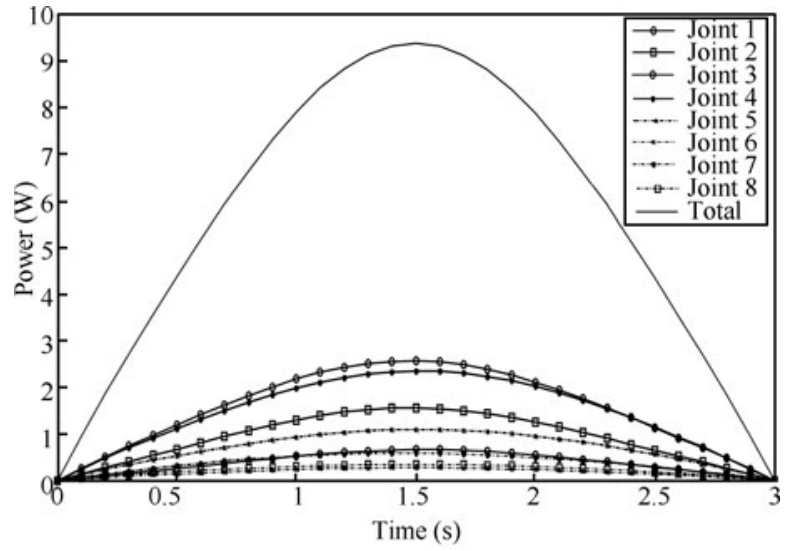

Fig. 5 Power consumptions for serpentine motion

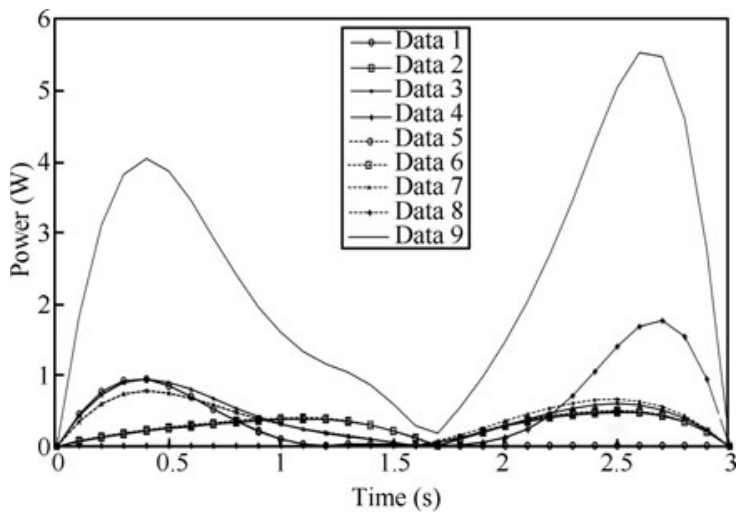

Fig. 6 Power consumptions for wriggler motion

Fig. 7 illustrates the gait switching algorithm developed and Fig. 8 shows flowchart of the algorithm implemented in this study. A new gait starts when all the data have been compared and the robot is in a new gait initial position. The simplest form of gait transition is to change all current joint angle values with a new initial position of the upcoming gait before a full gait sequence is executed. Through this approach, the gait kinematic structure can be guaranteed. The process is executed sequentially from the head down to the tail of the snake robot. The joint position only changes if the value between the two gaits is different, or else it remains the same. This change should be fast enough to avoid the robot from stop moving which could reduce the speed of locomotion.

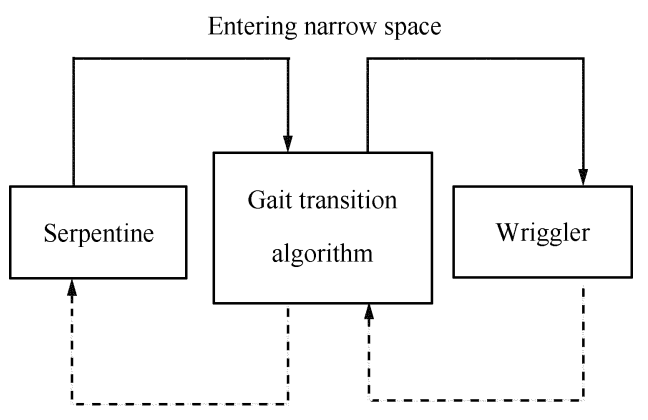

Entering open space

Fig. 7 Gait switching mechanism

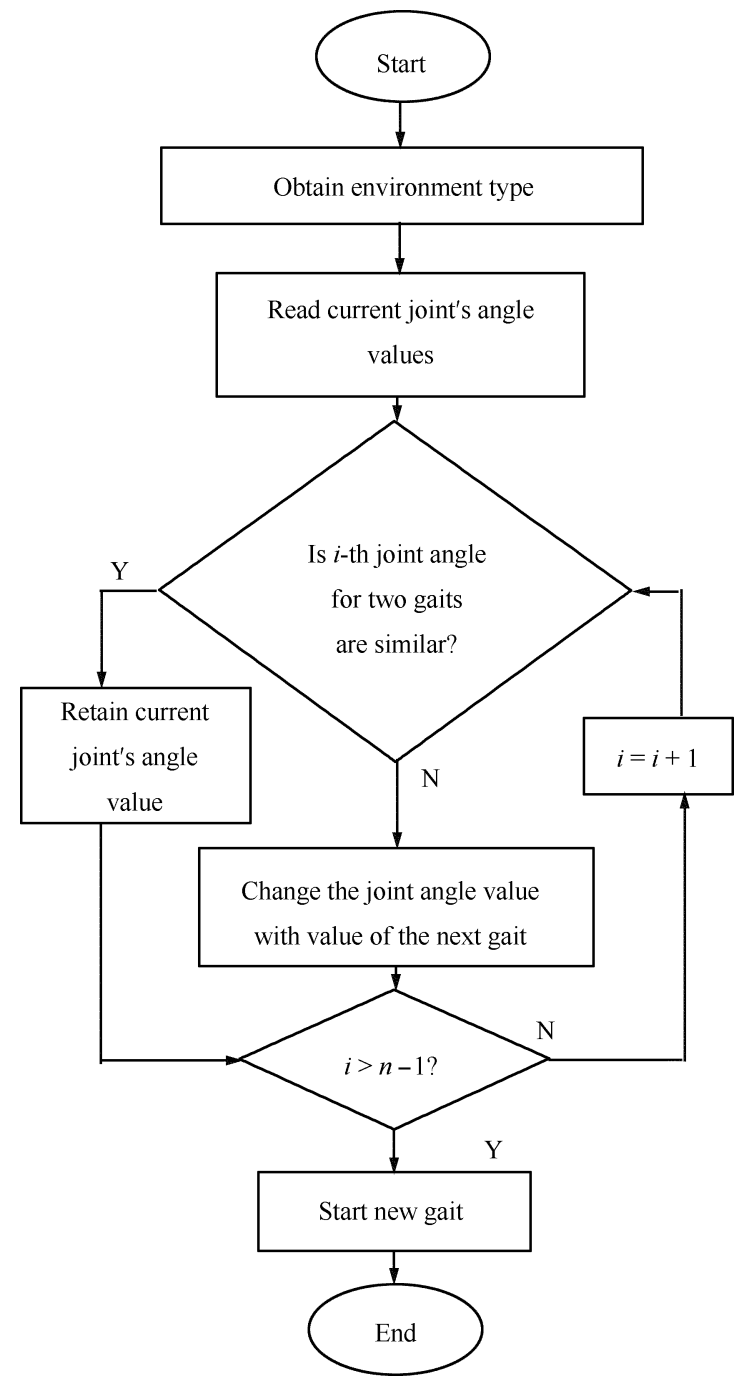

Fig. 8 Gait transition algorithm

In order to make the snake robot be able to choose correct mode of locomotion, a sensing system is required to analyse the working environment. For efficient sensing, the head of the snake robot should move parallel to the locomotion direction. In this research, a method known as head-navigation locomotion presented in [31] is considered to ensure the snake robot head move parallel to the direction of motion during serpentine locomotion. From Fig. 2, this statement means the absolute angle of the head $\theta_{n}$ is always zero. Fig. 9 shows the serpentine locomotion with head-navigation locomotion. However, for the wriggler gait its nature of its kinematics guarantees the movement of the head in parallel with the direction of motion.

Based on definition of the open space and narrow space given in Section 2, the diameter hull for serpentine gait is determined to be $30 \mathrm{~cm}$ while for wriggler gait is $10 \mathrm{~cm}$. Three range sensors are used to differentiate between the open space and narrow space and the positions of the sensors on the snake robot head are shown in Fig. 10.

Two sensors S1 and S2 are positioned at an angle $\rho$ between each other while S3 is placed parallel to the direction of motion. Sensor S1 is used to determine length of $l_{s 1}$ while 
sensor S2 is used to determine length $l_{s 2}$ as illustrated in Fig. 11. Once $l_{s 1}$ and $l_{s 2}$ are known, the diameter of the narrow space $d_{n}$ is determined by using the cosine law

$$
d_{n}=\sqrt{l_{s 1}^{2}+l_{s 2}^{2}-2 l_{s 2} \cos (\rho)} .
$$

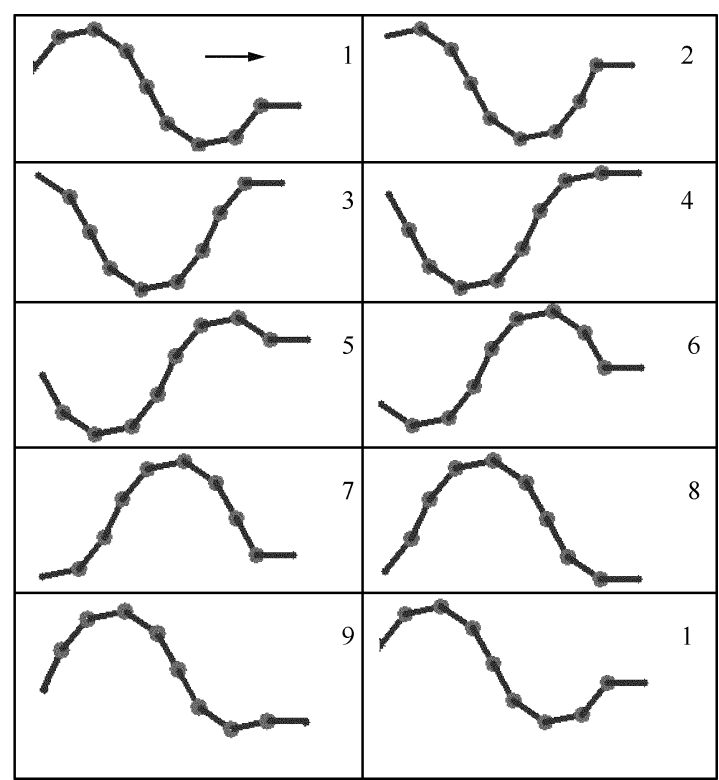

Fig. 9 Snake robot with head navigated locomotion direction of motion during a cycle of serpentine gait

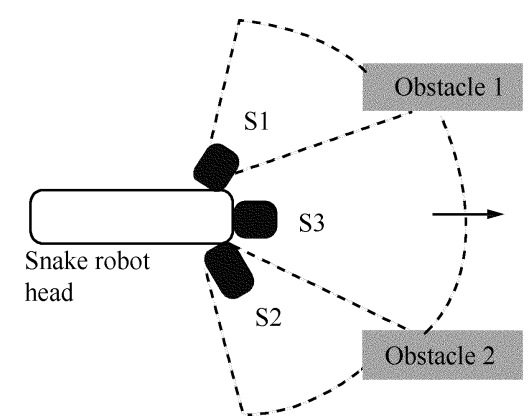

Fig. 10 Position of the sensor on the head of the snake robot

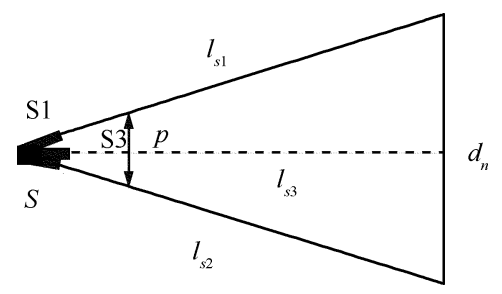

Fig. 11 Trigonometric for narrow space diameter computation

However, if S1 and S3 are activated then the diameter of the narrow space are given as

$$
d_{n}=\sqrt{l_{s 1}^{2}+l_{s 3}^{2}-2 l_{s 1} l_{s 3} \cos \left(\frac{\rho}{2}\right)} .
$$

Similar equation is used in case if S2 and S3 are activated, except that $l_{s 2}$ in (31) is replaced by $l_{s 3}$. If $d_{n}>30 \mathrm{~cm}$, serpentine gait is used for locomotion while if $10 \mathrm{~cm} \leqslant d_{n} \leqslant 30 \mathrm{~cm}$, wriggler gait is used to navigate. To make sure that the snake robot switches the gait just before entering the narrow space range, detection range are set low.

In this research, the range of the sensor is $4 \mathrm{~cm}$ to $30 \mathrm{~cm}$. Thus, the snake robot will switch its gait within 4 to $30 \mathrm{~cm}$ distance from the narrow space. However, from the manufacturer datasheet, the best range is between $4 \mathrm{~cm}$ and $16 \mathrm{~cm}$. This range is used so that the snake robot does not switch its gait far away before entering the narrow space. The snake robot will switch the gait back to serpentine when all three sensors are deactivated or no obstacle is detected in the range. The disadvantage of this method is that the head of the snake robot must always be in the direction of locomotion. Slippage during locomotion could cause the sensing method to become inaccurate. For better improvement, a fusion sensing system using camera and range sensor could provide a better result.

\section{Prototype design}

Development of the snake robot prototype requires the analysis of kinematics and dynamical constraints of the snake robot. The goal is to synchronize the gaits under consideration, or else the prototype might fail to work properly. The design of the snake robot body should satisfy both geometrical shape requirements of the two gaits. Since locomotion of the snake robot locomotion is purely based on kinematics, a proper control method is developed to generate the required shape of locomotion.

\subsection{Prototype structure}

A complete prototype design to satisfy these constraints is shown in Fig. 12 where the shape of the robot body is designed to adapt to the two geometrical structures of the gaits being considered. The design of the snake robot prototype in this research is inspired from a real snake structure. The link or the bone of the snake robot is shown in Fig. 13 (a) and the shape itself inspired from the snake vertebra is shown in Fig. 13 (b). This shape is stable and reduces the overall weight of the prototype. Fig. 14 (a) shows a set of artificial scales used to generate friction and placed at the belly of the robot to assist the robot to propel forward. The design and arrangement of the scales follow the real snake scales shown in Fig. 14 (a). The angle between the scales could be increased to provide better grip on the surface.

The serpentine gait requires backward direction frictional force to be higher compared to the forward direction. At the same time it must be able to avoid side slippage by providing higher frictional force in the tangential direction of motion as compared to the of friction force in the normal direction. If this condition is not satisfied, the snake robot will move sidewise as opposed to move forward. The normal friction force $f_{n}$ plays an important role in preventing side slippage while backward friction force $f_{b}$ assists the robot to move forward as shown in Fig. 15 (a). The normal friction force is generated by the two edges of the scale which act like a passive wheel in preventing side slippage. 


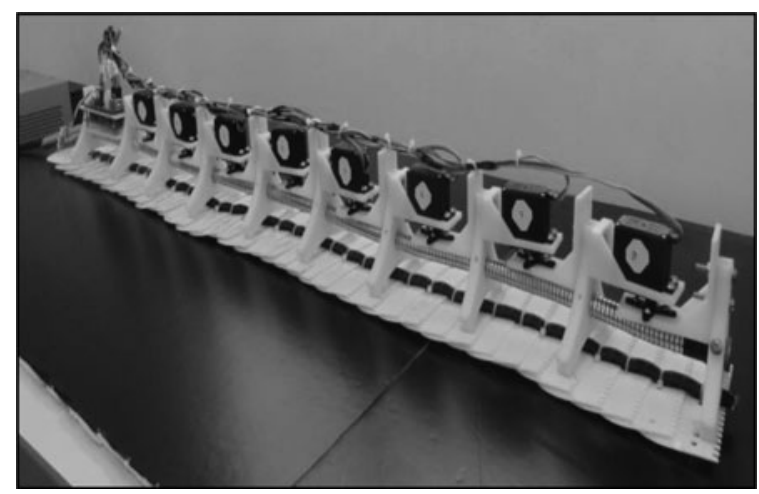

Fig. 12 Snake robot prototype

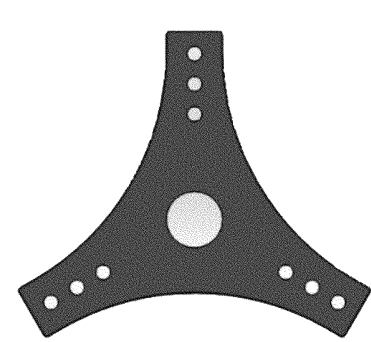

(a) Snake robot bone

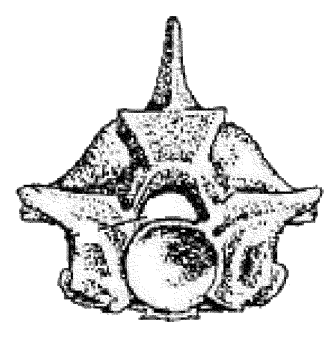

(b) Real snake vertebra
Fig. 13 Design concept of the snake robot bone

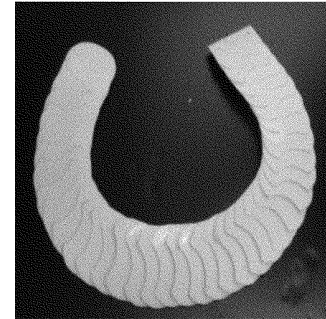

(a) Artificial scale

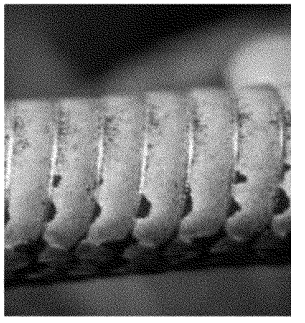

(b) Real snake scale
Fig. 14 Design concept of the snake robot scales

On the other hand, for wriggler gait to work effectively, the friction force is considered infinite and zero for backward and forward motions, respectively. In order to have the infinite backward friction force, the edge of the scale must dig-in into the ground and act as pin which allows only rotational motion at that point. The dig-in effect alternates between the two edges such that when one edge experiences the dig-in effect and the other is not. The mechanism is illustrated in Fig. 15 (b).

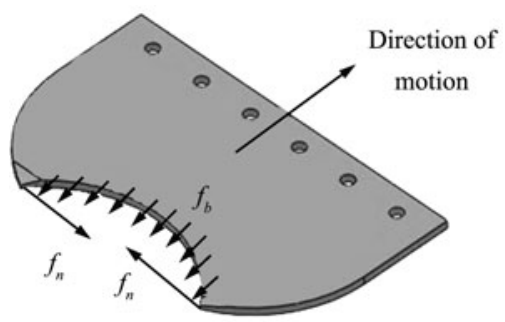

(a)

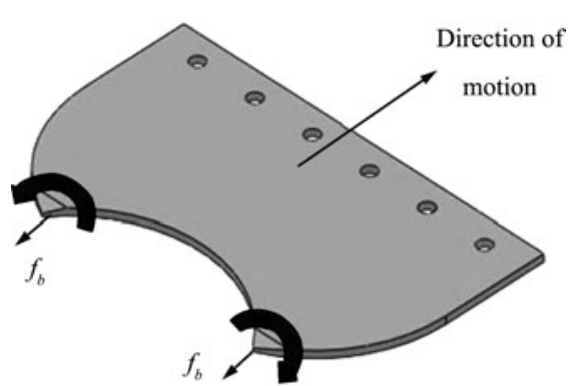

(b)

Fig. 15 Friction forces acting on the scales during locomotion. (a) Friction forces during serpentine locomotion; (b) Friction forces during wriggler locomotion

The scale is designed to have similar function as passive wheel (to avoid side slippage). The advantages of this design compared to passive wheels are that it provides better traction on the slope surfaces and prove to work for both gaits considered in this study. The nature of wriggler gait is not suitable to work with passive wheels.

However, from the experiment observation, larger slip displacement occurs when the snake robot is moving on the smooth surface with a low friction coefficient. The tendency to slip on smooth surface when moving with high speed reduces the speed of locomotion compared to the snake robot with wheels. In addition, our snake robot still lacks modularity because only one controller controls all the snake robot segments, compared to works that use master and slave concept of control.

\subsection{Control architecture}

The general control architecture of the snake robot is shown in Fig. 16. The required data to generate serpentine gait are obtained from (1) through (4) with parameters $a=\pi / 2, b=2 \pi$, and $c=0$ for straight line locomotion. Data for wriggler gait are calculated based on (18) through (20). The calculated data are then stored in a microcontroller in the form of arrays. The gait is generated by shifting the joint angles as illustrated in Fig. 17. In one cycle for both serpentine and wriggler gaits, there are eight steps for completing locomotion. The next steps are the repetition of the previous steps. To ensure the desired form of gait structure is generated, all the angles in each row must reach the desired position before continuing on to the next row, and the angles must arrive at the next configuration at the same time. Thus, it becomes clear that the speed of the servo motor must also be controlled. To arrive at the desired speed, absolute differences between each row are computed as shown in Table 2. Then, the largest value of the absolute difference associated with the highest controllable speed achievable by servo motor is used as the reference for adjusting speed of the other servos.

For example, in Table 2, the maximum absolute difference is between Steps 1 to 2 and is $50^{\circ}$. This maximum absolute value is then assigned with the maximum speed (in our case $353^{\circ} \mathrm{s}^{-1}$ ). The other values in the same row are assigned proportionally slower velocities such that if every servo is traversed according to the assigned speed then all 
the angles would arrive at their respective positions within the same amount of time. Continuing with this example, from the same row, since $4^{\circ}$ is 12.5 times smaller than the maximum of $50^{\circ}$, its speed would also be 12.5 times slower at $353^{\circ} \mathrm{s}^{-1}$ and the procedure is repeated for all elements in the row difference. The corresponding speeds could then be expressed as shown in Table 3 .

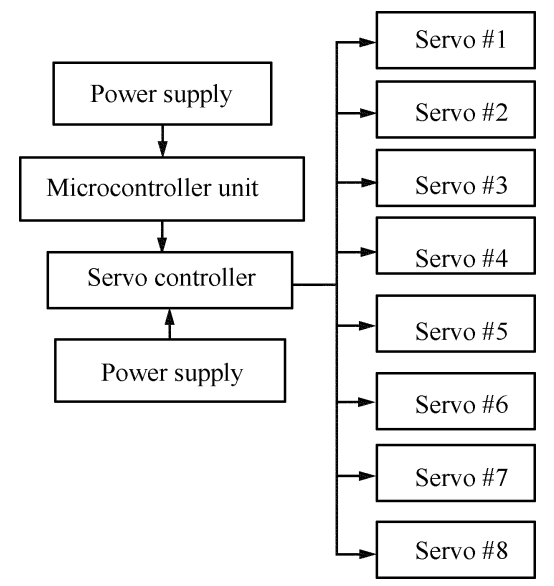

Fig. 16 General control architecture

\begin{tabular}{|c|l|l|l|l|l|l|l|l|}
\hline \multirow{2}{*}{ Step } & \multicolumn{7}{|c|}{ Joint } \\
\cline { 2 - 9 } & 1 & 2 & 3 & 4 & 5 & 6 & 7 & 8 \\
\hline 1 & $\phi_{1}$ & $\phi_{2}$ & $\phi_{3}$ & $\phi_{4}$ & $\phi_{5}$ & $\phi_{6}$ & $\phi_{7}$ & $\phi_{8}$ \\
\hline 2 & $\phi_{2}$ & $\phi_{3}$ & $\phi_{4}$ & $\phi_{5}$ & $\phi_{6}$ & $\phi_{7}$ & $\phi_{8}$ & $\phi_{2}$ \\
\hline 3 & $\phi_{3}$ & $\phi_{4}$ & $\phi_{5}$ & $\phi_{6}$ & $\phi_{7}$ & $\phi_{8}$ & $\phi_{2}$ & $\phi_{3}$ \\
\hline 4 & $\phi_{4}$ & $\phi_{5}$ & $\phi_{6}$ & $\phi_{7}$ & $\phi_{8}$ & $\phi_{3}$ & $\phi_{4}$ & $\phi_{5}$ \\
\hline 5 & $\phi_{5}$ & $\phi_{6}$ & $\phi_{7}$ & $\phi_{8}$ & $\phi_{4}$ & $\phi_{5}$ & $\phi_{6}$ & $\phi_{7}$ \\
\hline 6 & $\phi_{6}$ & $\phi_{7}$ & $\phi_{8}$ & $\phi_{5}$ & $\phi_{6}$ & $\phi_{7}$ & $\phi_{8}$ & $\phi_{4}$ \\
\hline 7 & $\phi_{7}$ & $\phi_{8}$ & $\phi_{6}$ & $\phi_{7}$ & $\phi_{8}$ & $\phi_{5}$ & $\phi_{6}$ & $\phi_{7}$ \\
\hline 8 & $\phi_{8}$ & $\phi_{7}$ & $\phi_{8}$ & $\phi_{6}$ & $\phi_{7}$ & $\phi_{8}$ & $\phi_{5}$ & $\phi_{6}$ \\
\hline
\end{tabular}

Fig. 17 Shifting of joint angle for gait generation in one cycle

Table 2 Joint's angle absolute difference

\begin{tabular}{ccccccccc}
\hline & \multicolumn{10}{c}{${\text { Join's angle }\left({ }^{\circ}\right)}$} \\
& $\varphi_{1}$ & $\varphi_{2}$ & $\varphi_{3}$ & $\varphi_{4}$ & $\varphi_{5}$ & $\varphi_{6}$ & $\varphi_{7}$ & $\varphi_{8}$ \\
\hline Step 1 & -36 & -40 & -26 & 0 & 26 & 40 & 36 & 14 \\
Step 2 & -40 & -26 & 0 & 26 & 40 & 36 & 14 & -36 \\
Absolute difference & 4 & 14 & 26 & 26 & 14 & 4 & 22 & 50 \\
\hline
\end{tabular}

Table 3 Association of speed with joint's angle absolute difference

\begin{tabular}{ccccccccc}
\hline & \multicolumn{10}{c}{ Join } \\
& $\phi_{1}$ & $\phi_{2}$ & $\phi_{3}$ & $\phi_{4}$ & $\phi_{5}$ & $\phi_{6}$ & $\phi_{7}$ & $\phi_{8}$ \\
\hline Absolute difference & 4 & 14 & 26 & 26 & 14 & 4 & 22 & 50 \\
Velocity $\left({ }^{\circ} \mathrm{s}^{-1}\right)$ & 28 & 99 & 184 & 184 & 99 & 28 & 155 & 353 \\
\hline
\end{tabular}

Since the microcontroller has a limited memory, combinations of the position and speed values of joints are necessary for storage. In this study, the servo controller has position values ranging from 0 to 1463 for $0^{\circ}$ to $180^{\circ}$ of rotation range, and speed range from 0 to 63 for $6^{\circ} \mathrm{s}^{-1}$ to $353^{\circ} \mathrm{s}^{-1}$. Thus position value of $36^{\circ}$ is converted to 1023 and the associated angular speed of $155^{\circ} \mathrm{s}^{-1}$ is converted to the equivalent value of 28 . Thus the combination value will be 102328 .

During the locomotion, the microcontroller routine will extract these combined values and then extracts the position and speed values before sending to the servo controller. The flow of the extraction process is shown in Fig. 18. For example, when a value of 102328 is called back by the routine of the microprocessor, it will first divide the number by 100 , arriving at 1023.28 and then truncate the decimal, leaving 1023. This value is stored temporarily as the new desired position for that particular servo motor.

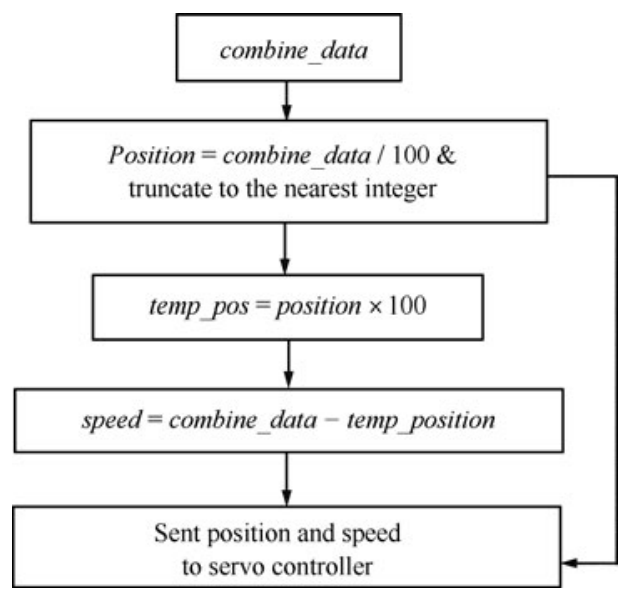

Fig. 18 Position and speed extraction routine

The same value would then be multiplied again by 100 to obtain 102300, and subtracted from the original value 102328, which is permanently stored, to extract the speed value which in this case is 28 . Using this method of extracting the two values from one stored element, a complete algorithm of a locomotion control based on kinematics is achievable.

\section{$7 \quad$ Experimental results}

A series of experiments have been conducted to test the viability of the proposed model and locomotion strategy. Table 4 summarizes the forward speeds achieved by the prototype under two different surfaces (i.e., carpet with higher friction and tiled floor with lower friction). These results demonstrate that the serpentine gait has a higher speeds compared to the wriggler gait for both surfaces as expected. Moreover, both gaits achieve higher speeds on the carpet (high friction) compared to the tiled floor (low friction). It also shows that the scales used in this study work better on a rough terrain compared to a smooth surface.

In terms of power consumption, the serpentine gait consumes more power compared to the wriggler gait as could be seen from Figs. 19 and 20. The power consumption is measured when the snake robot is moving on the carpet for 15 steps (1.5 cycles) of the motion. However, the values are larger than the simulated values due to actual friction effect at the contact surface. During the gait transition, average power consumption is less than the serpentine but higher 
than the wriggler as shown in Fig. 21, where transition occurred between $35 \mathrm{~s}$ to $60 \mathrm{~s}$.

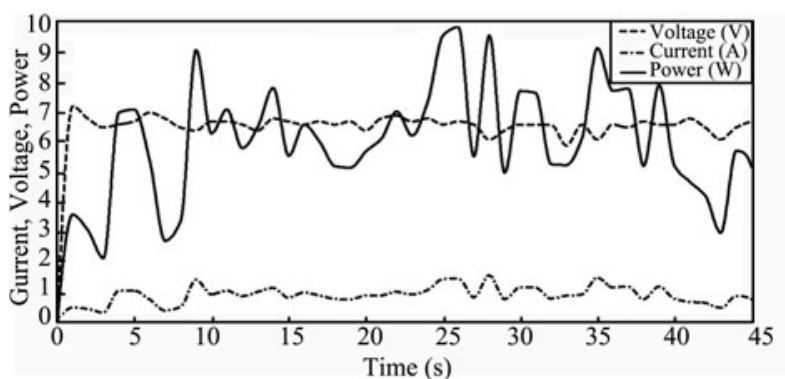

Fig. 19 Power consumption for wriggler gait

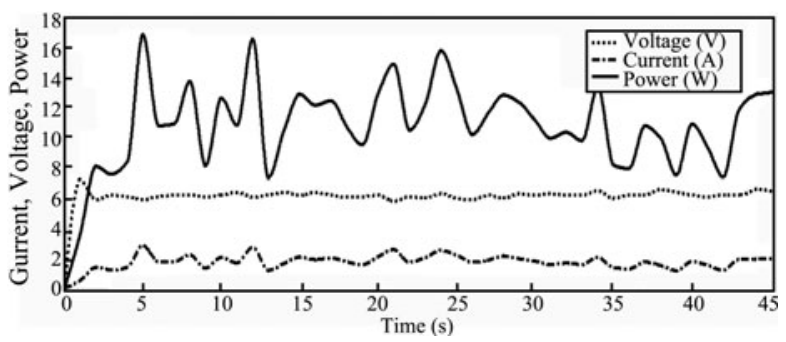

Fig. 20 Power consumption for serpentine gait

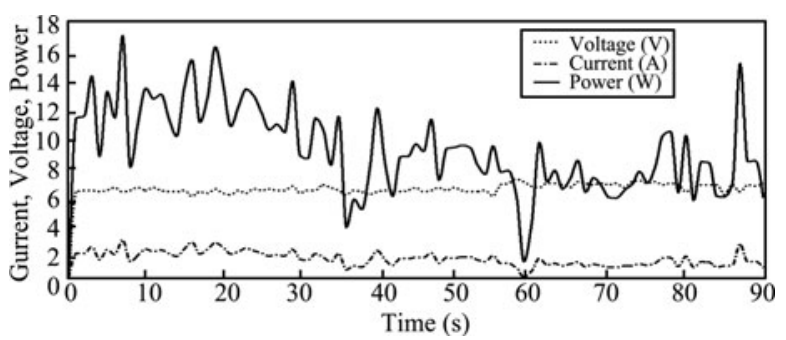

Fig. 21 Power consumption during gait transition form serpentine to wriggler

Fig. 22 shows snapshots of the snake robot passing trough a narrow passage by switching its gaits according to the environment. Starting with the serpentine gait in the open space in Fig. 22 (a), the snake robot switched its gait to the wriggler gait to enter a narrow passage in Fig. 22 (b). In the narrow passage, it executed full wriggler locomotion till it reached end of the passage as shown in Fig. 22 (c). Before entering the open space again, it switched back its locomotion gait to the serpentine in Fig. 22 (d) and finally propelled with full serpentine locomotion when its body was totally out of the narrow passage in Fig. 22 (e).

\section{Conclusions}

In this paper, a snake robot design using mixed gaits is described. A list of important considerations for the devel-
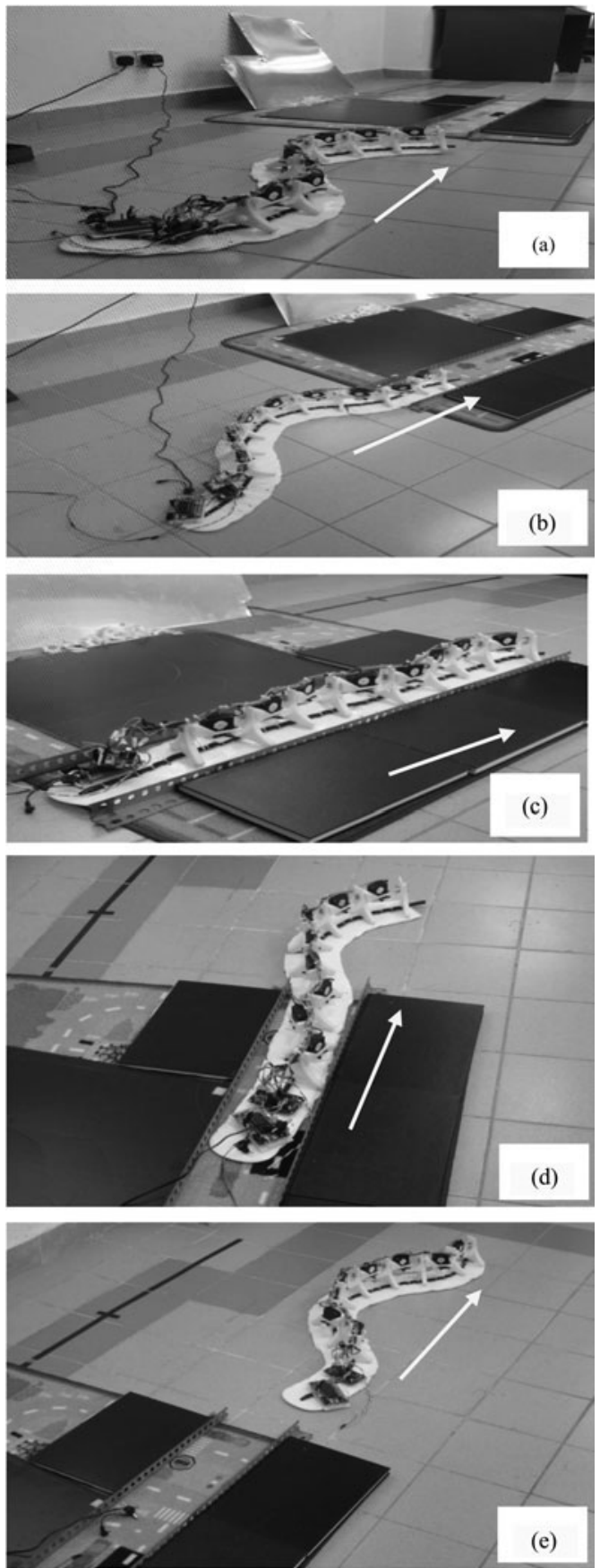

Fig. 22 Snake robot movement. (a) Open space-serpentine; (b) Transition from serpentine to wriggler; (c) Inside narrow spacewriggler; (d) Transition from wriggler to serpentine; (e) Open space-serpentine

Table 4 Forward speed for the snake robot prototype

\begin{tabular}{|c|c|c|c|c|c|c|c|c|}
\hline \multirow{3}{*}{ Testing environment } & \multicolumn{8}{|c|}{ Speed $(\mathrm{cm} / \mathrm{s})$} \\
\hline & \multicolumn{4}{|c|}{ Serpentine gait } & \multicolumn{4}{|c|}{ Wriggler gait } \\
\hline & Test 1 & Test 2 & Test 3 & Average & Test 1 & Test 2 & Test 3 & Average \\
\hline Carpet & 3.45 & 3.68 & 3.50 & 3.54 & 1.23 & 1.39 & 1.26 & 1.29 \\
\hline Tile floor & 2.12 & 2.40 & 2.18 & 2.23 & 1.03 & 1.17 & 1.21 & 1.14 \\
\hline
\end{tabular}


opment of the system is also highlighted. The kinematics for serpentine and wriggler gaits is described and the related geometrical constraints for respective gaits are also pointed out. The prototype developed for mixed mode gait has successfully executed the two gaits in two different workspaces. The result has also proved the efficacy of the proposed gait switching algorithm. In the future the 3D locomotion will be considered with turning capability for higher motion flexibility. Smart material will be considered to mimic snake muscle instead of using rigid actuator for better locomotion flexibility and environment adaptability.

\section{References}

[1] S. Hirose, Y. Umetani. Kinematic control of active cord mechanism with tactile sensors. In Proceedings of the 2nd RoMAnSy Symposium, RoMAnSy, Warsaw, Poland, pp. 241-252, 1976.

[2] J. Gray. The mechanism of locomotion in snakes. Journal of Experimental Biology, vol. 23, no. 2, pp. 101-120, 1946.

[3] S. Hirose. Biologically Inspired Robots: Snake-like Locomotors and Manipulators, Oxford, Lodon: Oxford University Press, 1993.

[4] K. H. Chang, Y. Y. Chen. Efficiency on snake robot locomotion with constant and variable bending angles. In Proceedings of IEEE Workshop on Advanced Robotics and Its Social Impacts, IEEE, Taipei, Taiwan, pp. 1-5, 2008.

[5] M. Dehghani, M. J. Mahjoob. A modified serpenoid equation for snake robots. In Proceedings of IEEE International Conference on Robotics and Biomimetics, IEEE, Bangkok, Thailand, pp. 1647-1652, 2009.

[6] G. Poi, C. Scarabeo, B. Allotta. Traveling wave locomotion hyper-redundant mobile robot. In Proceedings of the IEEE International Conference on Robotics and Automation, IEEE, Leuven, Belgium, pp. 418-423, 1998.

[7] J. W. Burdick, J. Radford, G. S. Chirikjian. A sidewinding locomotion gait for hyper-redundant robots. In Proceedings of IEEE International Conference on Robotics and Automation, IEEE, Atlanta, GA, USA, pp. 101-106, 1993.

[8] I. Tanev, T. Ray, A. Buller. Automated evolutionary design, robustness, and adaptation of sidewinding locomotion of a simulated snake-like robot. IEEE Transactions on Robotics, vol. 21, no. 4, pp. 632-645, 2005.

[9] R. L. Hatton, H. Choset. Sidewinding on slopes. In Proceedings of IEEE International Conference on Robotics and Automation, IEEE, Anchorage, Alaska, USA, pp. 691-696, 2010.

[10] M. Tanaka, F. Matsuno. Modeling control of a snake robot with switching constraints. In Proceedings of SICE Annual Conference, IEEE, Tokyo, Japan, pp. 3076-3079, 2008.

[11] C. Y. Liu, W. H. Liao. A snake robot using shape memory alloys. In Proceedings of IEEE International Conference on Robotics and Biomimetics, IEEE, Shenyang, China, pp. 601-605, 2004.

[12] B. W. Spranklin. Design, Analysis, and Fabrication of a Snake-inspired Robot with a Rectilinear Gait, Master dissertation, University of Maryland, USA, 2006.

[13] A. Gmiterko, I. Virgala. Simplified model of the snake rectilinear motion. In Proceedings of the 9th IEEE International Symposium on Applied Machine Intelligence and Informatics, IEEE, Smolenice, Slovakia, pp. 307-310, 2011.
[14] F. Barazandeh, B. Bahr, A. Moradi. Investigation of selflocking in concertina movement. In Proceedings of Mediterranean Conference on Control and Automation, IEEE, Athens, Greece, pp. 1-6, 2007.

[15] A. Akbarzadeh, J. Safehian, H. Kalani. Generating snake robot concertina locomotion using a new dynamic curve. International Journal of Modeling and Optimization, vol. 1, no 2, pp. 134-140, 2011.

[16] S. Hasanzadeh, A. A. Tootoonchi. Obstacle avoidance of snake robot moving with a novel gait using two-level PID controller. In Proceedings of IEEE Conference on Robotics, Automation and Mechatronics, IEEE, Chengdu, China, pp. 427-432, 2008.

[17] S. Hasanzadeh, A. A. Tootoonchi. Ground adaptive and optimized locomotion of snake robot moving with a novel gait. Autonomous Robots, vol. 28, no. 4, pp. 457-470, 2010.

[18] S. M. Yu, S. G. Ma, B. Li, Y. C. Wang. Analysis of helical gait of a snake-like robot. In Proceedings of IEEE/ASME International Conference on Advanced Intelligent Mechatronics, IEEE, Xian, China, pp. 1183-1188, 2008.

[19] H. Shin, K. M. Jeong, J. J. Kwon. Development of a snake robot moving in a small diameter pipe. In Proceedings of International Conference on Control, Automation and Systems, IEEE, Gyeonggi-do, Korea, pp. 1826-1829, 2010.

[20] T. Baba, Y. Kameyama, T. Kamegawa, A. Gofuku. A snake robot propelling inside of a pipe with helical rolling motion. In Proceedings of SICE Annual Conference, IEEE, Taipei, Taiwan, China, pp. 2319-2325, 2010.

[21] H. Yamada, S. Hirose. Steering of pedal wave of a snakelike robot by superposition of curvatures. In Proceedings of IEEE/RSJ International Conference on Intelligent Robots and Systems, IEEE, Taipei, Taiwan, China, pp. 419-424, 2010.

[22] W. M. Shen, M. Krivokon, H. Chiu, J. Everist, M. Rubenstein, J. Venkatesh. Multimode locomotion via SuperBot robots. In Proceedings of IEEE International Conference on Robotics and Automation, IEEE, Orlando, Florida, USA, pp. 2252-2257, 2006.

[23] A. Crespi, A. J. Ijspeert. Online optimization of swimming and crawling in an amphibious snake robot. IEEE Transactions on Robotics, vol. 24, no. 1, pp. 75-87, 2008.

[24] K. D. Wang, S. G. Ma. Kinematic analysis of snake-like robot using sliding joints. In Proceedings of IEEE International Conference on Robotics and Biomimetics, IEEE, Tianjin, China, pp. 1484-1489, 2010.

[25] J. Zhao, X. D. Cui, Y. H. Zhu, S. F. Tang. A new self-reconfigurable modular robotic system UBot: Multimode locomotion and self-reconfiguration. In Proceedings of IEEE International Conference on Robotics and Automation, IEEE, Shanghai, China, pp. 1020-1025, 2011.

[26] M. Yim, D. G. Duff, K. D. Roufas. PolyBot: A modular reconfigurable robot. In Proceedings of 2000 IEEE International Conference on Robotics and Automation, IEEE, San Francisco, CA, USA, pp. 514-520, 2000.

[27] N. Li, T. S. Zhao. A snake-like robot based on a spatial linkage mechanism and its kinematics. In Proceedings of 2007 IEEE International Conference on Robotics and Biomimetics, IEEE, Sanya, China, pp. 995-1000, 2007.

[28] M. Saito, M. Fukaya, T. Iwasaki. Serpentine locomotion with robotic snakes. IEEE Control Systems Magazine, vol. 22 , no. 1, pp. 64-81, 2002. 
[29] R. Khan, M. Watanabe, A. A. Shafie. Kinematics model of snake robot considering snake scale. American Journal of Applied Sciences, vol. 7, no. 5, pp. 669-674, 2010.

[30] S. B. Niku. Introduction to Robotics: Analysis, Systems, Applications, New Jersey, USA: Prentice Hall, pp. 120-133, 2001.

[31] X. D. Wu, S. G. Ma. Head-navigated locomotion of a snake-like robot for its autonomous obstacle avoidance. In Proceedings of 2010 IEEE/RSJ International Conference on Intelligent Robots and Systems, IEEE, Taipei, Taiwan, pp. 401-406, 2010.

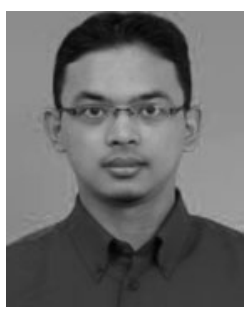

M. H. A. Majid received his B. Sc. and M. Sc. degrees in mechatronics engineering from the International Islamic University Malaysia, Malaysia in 2009 and 2012, respectively. His thesis title was "Design and development of a snake robot with mixed mode locomotion". Currently, he is a research assistant at the Department of Mechatronics Engineering, International Islamic University, Malaysia.

His research interests include biomimetic robotics, intelligent system, mechatronics system design and feedback control systems.

E-mail: helmi_mjd@yahoo.com

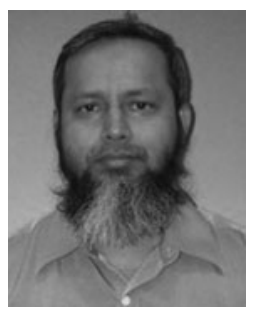

M. R. Khan received his B.Sc. degree in mechanical engineering from Rajshah University of Engineering and Technology, Bangladesh in 1983. He received M.Sc. and $\mathrm{Ph} . \mathrm{D}$. degrees in mechanical engineering from the Bangladesh University of Engineering and Technology, Bangladesh in 1988 and 1996, respectively. Currently, he is an associate professor in the Department of Mechatronics Engineering, International Islamic University, Malaysia.

His research interests include robotics, stress and stability of structures, smart structures and active vibration control. He is a member of IEEE.

E-mail: raisuddin@iium.edu.my (Corresponding author)

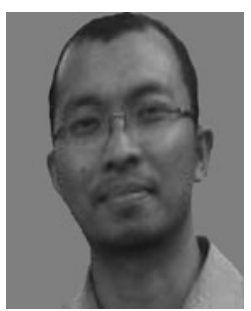

S. N. Sidek received the B. Eng. and $\mathrm{Ph}$. D. degrees from Vanderbilt University in 1998 and 2008, respectively. Currently, he serves as associate professor of Mechatronics Engineering at Department of Mechatronics Engineering, International Islamic University Malaysia. $\mathrm{He}$ is currently a senior member of IEEE.

His research interests include humancentered electromechanical system, human robot interaction and affective state computing.

E-mail: snaim@iium.edu.my 